\title{
Design for a Micro Strip Line Fed I Shaped Dielectric Resonator Antenna for Milimeter Wave Application
}

\author{
Arun Agarwal ${ }^{1, *}$, Kabita Agarwal $^{2}$, Gourav Misra ${ }^{3}$ \\ ${ }^{1}$ Department of Electronics and Communication Engineering, Institute of Technical Education \& Research, \\ Siksha 'O' Anusandhan Deemed to be University, Khandagiri Square, Bhubaneswar-751030, Odisha, India \\ ${ }^{2}$ Department of Electronics and Telecommunication Engineering, CV Raman College of Engineering, \\ Bhubaneswar-752054, Odisha, India \\ ${ }^{3}$ School of Electronic Engineering, Dublin City University, Glasnevin, Dublin 9, Ireland \\ *Corresponding author: arunagrawal@soa.ac.in
}

Received June 10, 2019; Revised July 13, 2019; Accepted July 26, 2019

\begin{abstract}
Requirement of antenna is essential in communication to transfer signals for various application. Different frequency range like the WLAN, WiMax, Ku-band, K-band, Ka-band, etc. in the world of communication has its arrangement. Microwave and satellite communication need higher range of frequencies for operation. Here, we have modelled a multi-band micro strip antenna for Ka band $(26.5 \mathrm{GHz}-40 \mathrm{GHz})$. The modelled antenna is simulated in Computer Simulated Technology. We observed that VSWR of the proposed antenna is below $2 \mathrm{~dB}$ in entire frequency range of $30.8 \mathrm{GHz}$ to $39 \mathrm{GHz}$. The designed antenna is simulated and a return loss of $-10 \mathrm{db}$ result is found out, which is in a range of $26.5 \mathrm{GHz}-40 \mathrm{GHz}$.
\end{abstract}

Keywords: WLAN, WIMAX, Ka band, modelled antenna, VSWR

Cite This Article: Arun Agarwal, Kabita Agarwal, and Gourav Misra, "Design for a Micro Strip Line Fed I Shaped Dielectric Resonator Antenna for Milimeter Wave Application." American Journal of Electrical and Electronic Engineering, vol. 7, no. 2 (2019): 49-54. doi: 10.12691/ajeee-7-2-5.

\section{Introduction}

\subsection{Motivation}

Satellite communication and wireless communication has been developing rapidly in the past decades. Also, it has been as of now an emotional effect on human life. In the couple of years, the advancement of remote neighborhood (WLAN) spoke to one of the key interests in the data and correspondence felid. The urgent segment of remote system or gadgets is radio wire. Very soon, our urban communities will be overflowed with receiving wire which has high proficient, low profile and little estimated .so dielectric resonator reception apparatus has gained an incredible ground in late year, as contrasted and customary radio wires. DRA have qualities like minimal effort and low profile which ends up being appropriate for WLAN/WI-MAX applications frameworks. The primary inspiration of this work is presently day, mobile correspondence frameworks are ending up progressively popular. Antennas for programming characterized and reconfigurable radio frameworks need ultra-wide band or multi-band attributes so as to be sufficiently adaptable to cover any conceivable future versatile correspondence recurrence bands. One way to deal with give such adaptability is to be build multiband reception apparatus that works over explicit narrowband frequencies. However, it would be very hard to precisely accomplish the recurrence prerequisites of all future correspondence system. Alternatively, a little wide band receiving wire that covers a wide scope of frequencies can be a decent hopeful for flow multi-band applications as well as for future correspondence frameworks working on new recurrence groups with data transmissions.

Requirements for the computerized home incorporate rapid information exchange for mixed media content, short-territory availability for exchange to different gadget

- Satellite correspondence framework

- Radar and imaging

- Medical applications

- Location and following

- Low information rate and low power UWB correspondences.

\subsection{Design Goals}

\subsubsection{Purpose}

As Satellite technology is developing enormously, designs has been developed for various satellite frequency band which is not only used for radio applications but also can be used in an astronomical applications, weather forecasting, broadcast, mapping, and many other applications.

With the increase in number of satellites, congestion has become a serious issue in the lower frequency ranges .So, new technologies are being discovered so that higher frequency bands can be used.

The higher frequency range for satellite communications is called the Ka-band which lies between $26.5 \mathrm{GHz}$ to 
40.0GHz. Ka-band is being primarily used for satellite communications, mainly as the downlink used by direct broadcast satellites to broadcast satellite television, and for specific applications.

\subsubsection{Scope}

Millimeter wave dielectric resonator antenna can for the application of $5 \mathrm{G}$ network connection in future.

\subsubsection{Applicability}

This type of antenna has been used and designed to operate in the $\mathrm{Ka}$ band which is used for satellite communication.

\subsection{Problem Statement}

To design a dielectric resonator antenna that will very small in size, light in weight and easy for installation. We have designed dielectric resonator antenna for Ka-band applications that can be used in satellite, aircraft and military applications. This antenna is designed using CST MWS software.

\section{Literature Survey}

\subsection{Project Specification}

We have executed the dielectric resonator receiving wire utilizing CST programming. An arrival loss of $-10.0 \mathrm{db}$ is found which is in the scope of $26.50 \mathrm{GHz}$ to $40 \mathrm{GHz}$. In this structure of data transfer capacity improvement of $24.36 \%$ has been achieved. From Figure 2 VSWR of the DRA is underneath 2 in the whole recurrence run $30.58 \mathrm{GHz}$ to $39 \mathrm{GHz}$. This arrival misfortune speaks to how much influence is reflected from the radio wires and subsequently is known as the reflection coefficient. The VSWR is a surely understood sign of how great the impedance coordinate is. A high VSWR means that the flag is reflected preceding being emanated by the antenna. The increase of receiving wires is $10.9 \mathrm{dBi}$.Antennae gain depicts how much power is transmitted in the pinnacle course radiation to that of isotropic source. Directivity of reception apparatuses is $10.24 \mathrm{dbi}$ at $35.46 \mathrm{GHZ}$ recurrence range. Directivity is the proportion of how directional a radio wires' radiation design is.

A dielectric resonator antenna usually is an antenna fabricated used at microwave frequencies and higher. A dielectric resonator antenna consists of a patch of ceramic material of various shapes. It is placed on a metallic surface known as ground plane. Common microstrip antenna shapes are square, rectangular, cylindrical and elliptical.

Dielectric resonator antennas have become very popular in recent decades due to their its high efficiency which can be incorporated into the surfaces of consumer products, aircraft and satellite, their ease of fabrication and the advantage of fractional bandwidth makes them active antenna.

Among the various types of antenna used today, satellite antennas are in a huge demand on this date. As the scientists want to know more and more about the world outside earth, we need more and more satellite antennas for better communication. This modelled antenna which works in the ka-band can be used for various purposes.

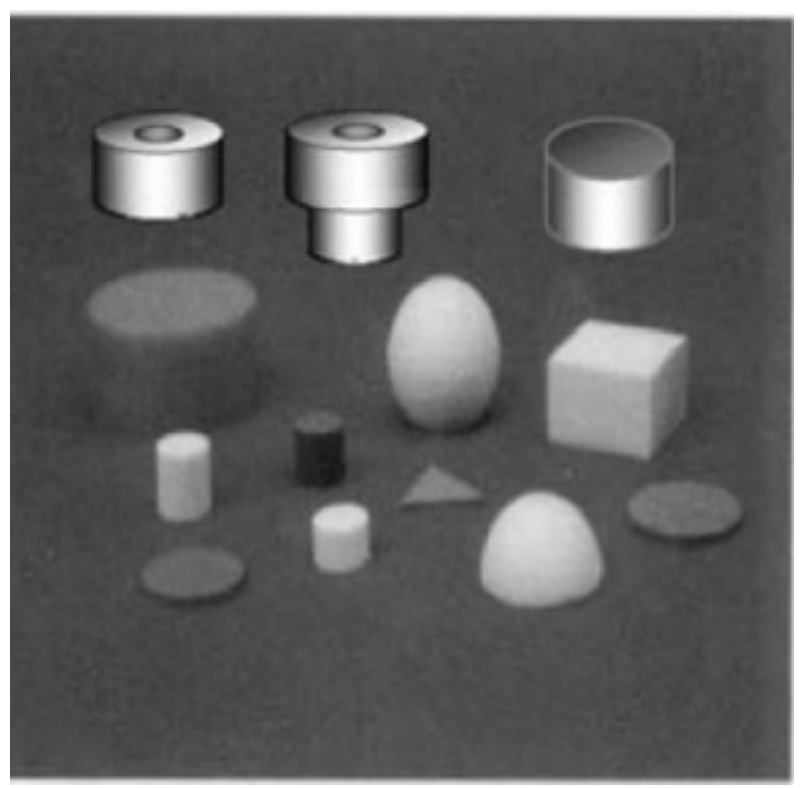

Figure 1. Different shapes of dielectric resonator antenna

\section{Design Scheme}

\subsection{System Design}

Dielectric resonator antennas have become very popular in recent decades due to their thin planar profile which can be incorporated into the surfaces of consumer products, aircraft and microwave filter. The basic design of a Dielectric resonator antenna constitutes

Ground plane: serves as a reflecting surface for radio waves. The ground plane also increases the directivity and maintains the radiating pattern.

Dielectric Substrate: Substrate is a base on which dielectric resonator antenna is fabricated and it plays important role in the antenna's functioning. With the increase in thickness of the substrate, the fringing increases which decreases the resonating frequency of the antenna (the fringing fields produce the radiation of the dielectric resonator antenna).Substrate (dielectrics) are used for improved electrical and mechanical stability. Hence, a substrate can enhance antenna's radiation capability.

Patch: Patch is responsible for radiation. Slots in the patch enable us to design dielectric resonator antenna with improved bandwidth and efficiency. The bandwidth and the resonating frequency of the antenna depends on the size of the slots and also on the distance between them.

\section{Line feeding technique:}

There are several techniques available to feed or transmit the electromagnetic energy to a dielectric resonator antenna.

The four most popular feed techniques used are:

- microstrip line (contacting scheme)

- coaxial probe (both contacting scheme)

- aperture coupling (non-contacting scheme)

- proximity coupling (non-contacting scheme). 

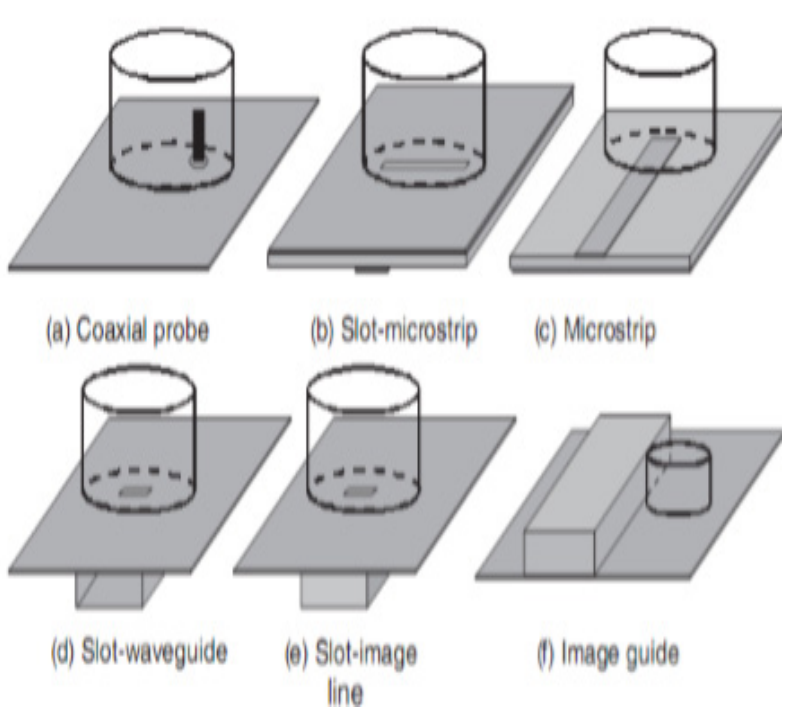

Figure 2. Different excitation mechanism for DRA

\section{FEEDING TECHNIQUES:}
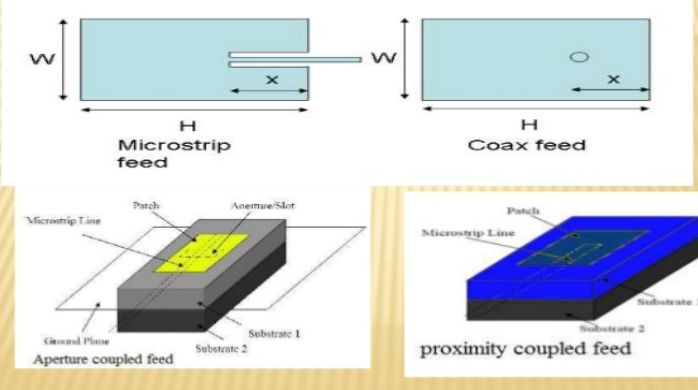

Figure 3. Feeding techniques of a microstrip patch antenna
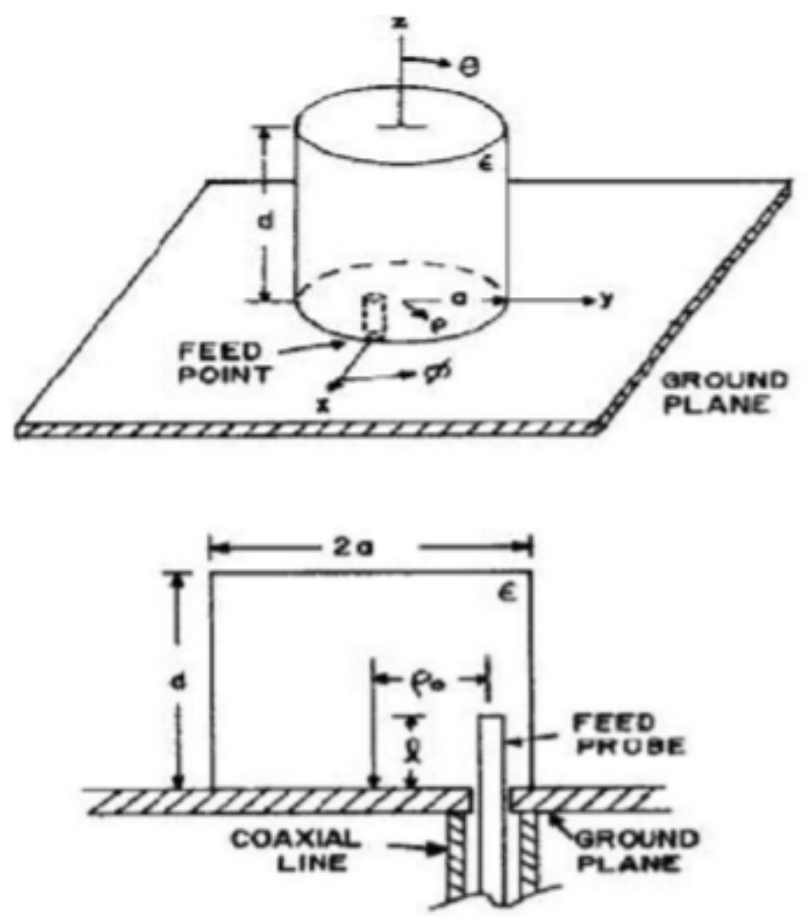

Figure 4. Geometry of cylindrical DRA

Microstrip line feed is a conducting strip is connected directly to the edge of the microstrip patch. The conducting strip is smaller in width as compared to the patch. This kind of feed arrangement has the advantage that the feed can be etched on the same substrate to provide a planar structure. An inset cut can be incorporated into the patch in order to obtain good impedance matching without the need for any additional matching element. It provides ease of fabrication and simplicity in modeling as well as impedance matching.

Coaxial probe: The main advantage of this type of feeding scheme is that the feed can be placed at any desired position inside the patch in order to obtain impedance matching. This feed method is easy to fabricate and has low spurious radiation effects. However, its major disadvantage is that it provides narrow bandwidth and is difficult to model since a hole has to be drilled into the substrate.

Aperture coupling: It is difficult to fabricate due to multiple layers, which also increases the antenna thickness.

Proximity coupling: the feed line is between the two substrates and the radiating patch is on top of the upper substrate.

\subsection{Architecture}

For huge numbers of the current and developing correspondence applications, wide band radio wire activity is alluring to suit the expanding information rates required for administrations, for example, video conferencing, direct computerized broadcast, EHF versatile satellite interchanges, nearby multipoint interchanges, and indoor remote. A portion of these necessities might be met by existing printed radio wire innovation, yet with the additional expense and unpredictability related with multi-layer arrangements required for accomplishing wide data transmissions. This area shows some novel DRAs of moderately basic design, which has shown wide-band execution, and may fill in as reasonable reception apparatus contender for these different applications.

The dielectric resonator antenna constitutes a ground plane, a substrate, a patch and a feeding technique .We can also slot the patch if required.

Width of the patch:

$$
W_{t}=\frac{C}{2 f_{0} \sqrt{\frac{\left(\epsilon_{r t}+1\right)}{2}}}
$$

Effective Dielectric Constant:

$$
\epsilon_{r e f f t}=\frac{\left(\epsilon_{r t}+1\right)}{2}+\frac{\left(\epsilon_{r t}-1\right)}{2}\left[1+12 \frac{h_{t}}{w_{t}}\right]^{\frac{1}{2}}
$$

Effective Length Calculation:

$$
l_{\text {eff } t}=\frac{c}{2 f_{o} \sqrt{\epsilon_{\text {reff } t}}}
$$

Extension Of Length:

$$
\Delta L_{t}=0.412 h_{t} \frac{\left(\epsilon_{\text {reff } t}+0.3\right)\left(\frac{w_{t}}{h_{t}}+0.264\right)}{\left(\epsilon_{\text {reff } t}-0.258\right)\left(\frac{w_{t}}{h_{t}}+0.8\right)}
$$


Length Of The Patch:

$$
L_{t}=L_{e f f_{t}}-2 \Delta
$$

Ground Plane Length and Width:

$$
\begin{aligned}
& L_{g}=6 h_{t}+L_{t} \\
& w_{g}=6 h_{t}+w_{t}
\end{aligned}
$$

Input Impedance:

$$
Z_{\text {in }}=90 \frac{2 \epsilon_{r}}{\epsilon_{r}-1}\left[\frac{L}{W}\right]^{2}
$$

\subsection{Component Design}

The antenna can be easily used as copper, silver, gold, etc. Here we choose copper because the material is cheap, it has a high conductivity and is easy.

The substrate is a layer between the ground and the patch. The properties of the substrates as dielectric constant, such as tangent of losses, affect the characteristics of this antenna. As we are designing a high frequency antenna, it is better to take the FR-4 substrate.

An antenna patch is made up of copper, which is very conductive, flexible and ductile. Malachite properties square, rectangular, cylindrical, elliptical, etc.

\subsection{Implementation}

The designed antenna has the following dimensions:

\begin{tabular}{ccc}
\hline S1. No. & Parameters & Dimensions $(\mathrm{mm})$ \\
\hline 1. & Ground & $15^{*} 25$ \\
2. & Substrate & $15^{*} 25^{*} 1.5$ \\
3. & Patch & $16^{*} 14$ \\
4. & Slot Patch & $5^{*} 1$ \\
5. & Microstrip Line & $8.5 * 1$ \\
\hline
\end{tabular}

\subsection{Design Evolution}

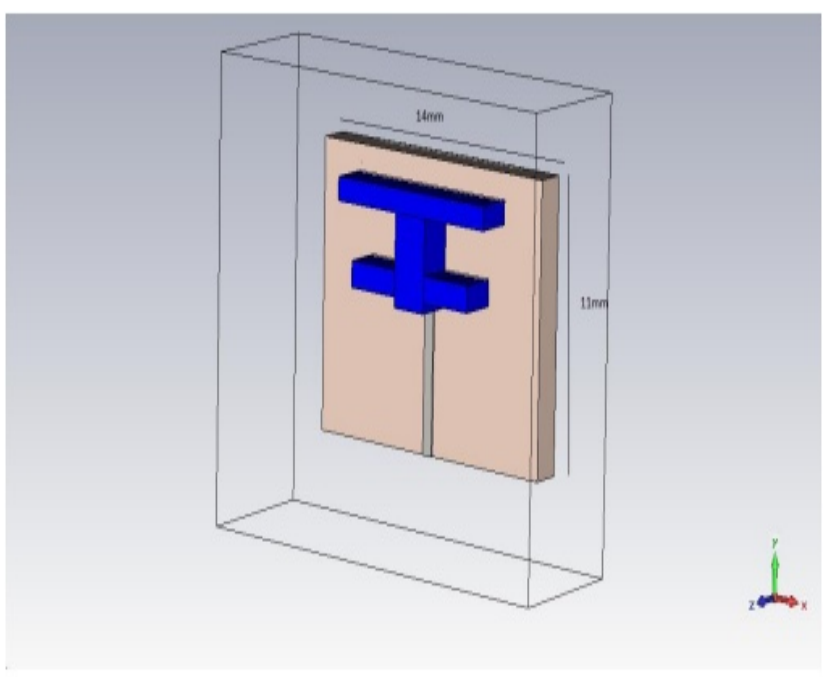

Figure-5. Geometry of proposed I shaped DRA

The simulated antenna is simulated and below $-10 \mathrm{~dB}$ return loss is achieved between $30.58 \mathrm{GHz}$ and $39 \mathrm{GHz}$.
The design antenna can be used to apply satellite communications, radio positioning and weapons and detection of dangerous materials. This design is for the $\mathrm{Ka}$ team $(26.5-40 \mathrm{GHz})$. In satellite communications, the bandwidth offers more bandwidth. The simulated returnloss loss of I-shaped D is represented by the frequency shown in the figure.

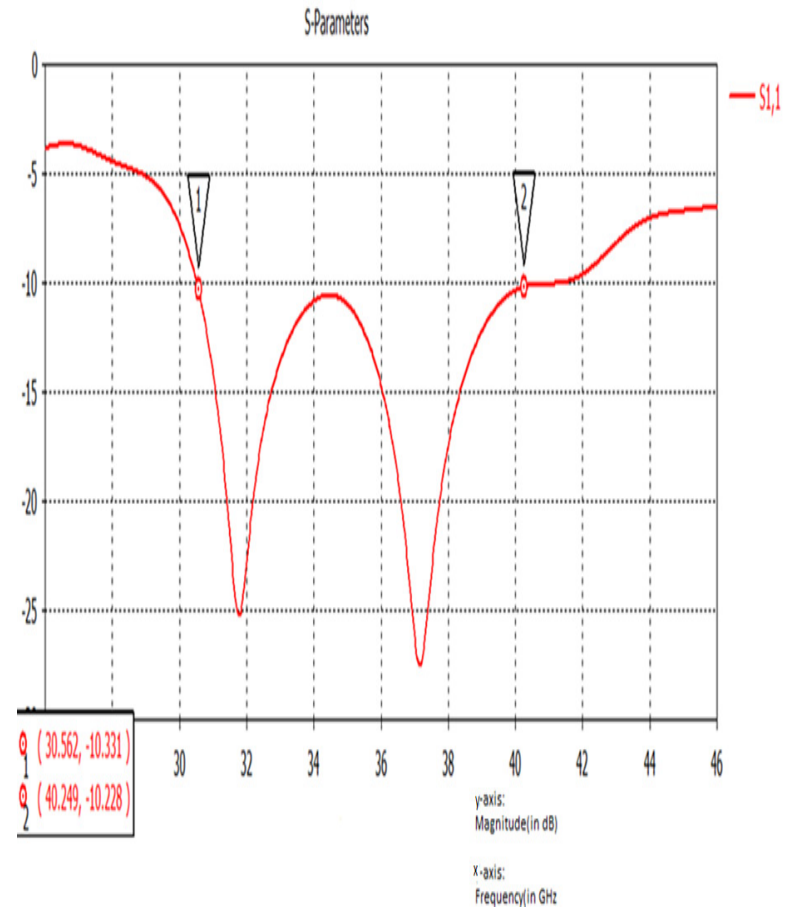

Figure 6. Simulated Reflection coefficient (Return loss) for the I-shaped DRA versus frequency

\section{Voltage Standing Wave Ratio (VSWR):-}

The voltage standing wave ratio (VSWR) is how good impedance match is. The VSWR of the proposed antenna is below in the frequency range of $30.58 \mathrm{GHz}$ to $40 \mathrm{GHz}$.

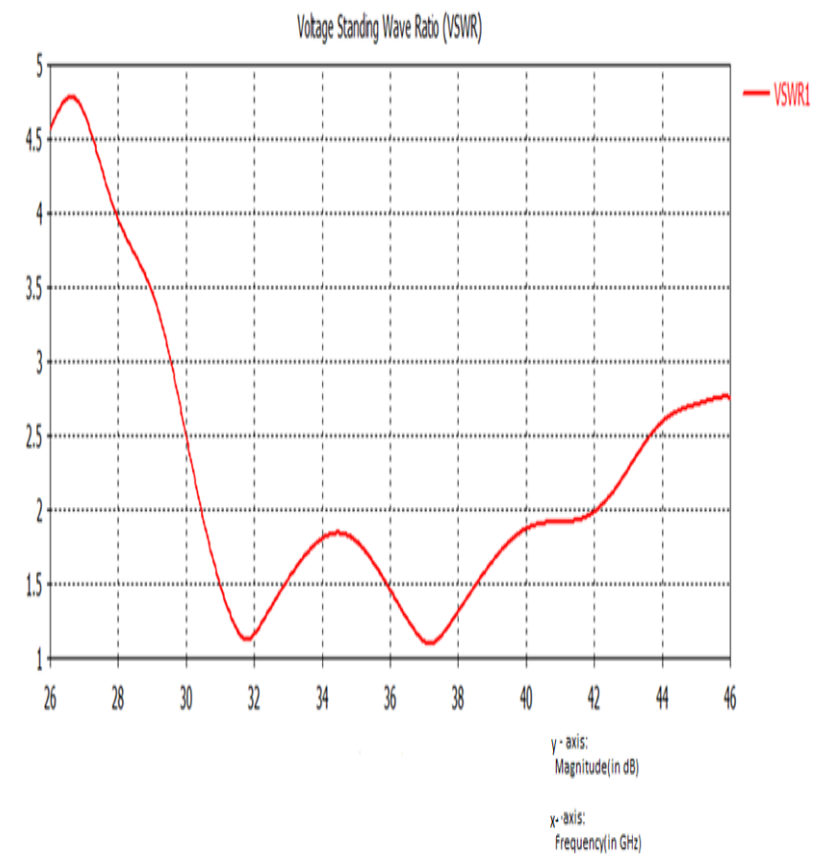

Figure 7. Simulated VSWR for the I-shaped DRA antenna 


\section{Testing, Analysis and Evaluation}

\subsection{Testing Criterions}

The proposed antenna is simulated and the loss of the $-10 \mathrm{db}$ return is achieved between $30.58 \mathrm{GHz}$ and $39 \mathrm{GHz}$. There is a good loss of return with the value of both devices $-21 \mathrm{db}$ to $31.54 \mathrm{GHz}$ and $-22 \mathrm{db}$ value. $35.39 \mathrm{GHz}$. In this design, $24.36 \%$ broadband improvement has been achieved. In addition, the efficiency of $72.88 \%$ is $35.39 \mathrm{GHz}$ and $62 \% 31.54 \mathrm{GHz}$. The design is aimed at the bandwidth (26.5-40 GHz). In satellite communications, the band has broadband communications. It allows relieving signal conditions in rain. This design, especially for satellites UPLINK frequencies $(31 \mathrm{GHz})$. Communication from the Uplink terrestrial terminal to a satellite communication connection ("aerotransport platform") to a satellite. The frequent I-shape is shown in the simulated image of the loss of return proposed by the DRA.

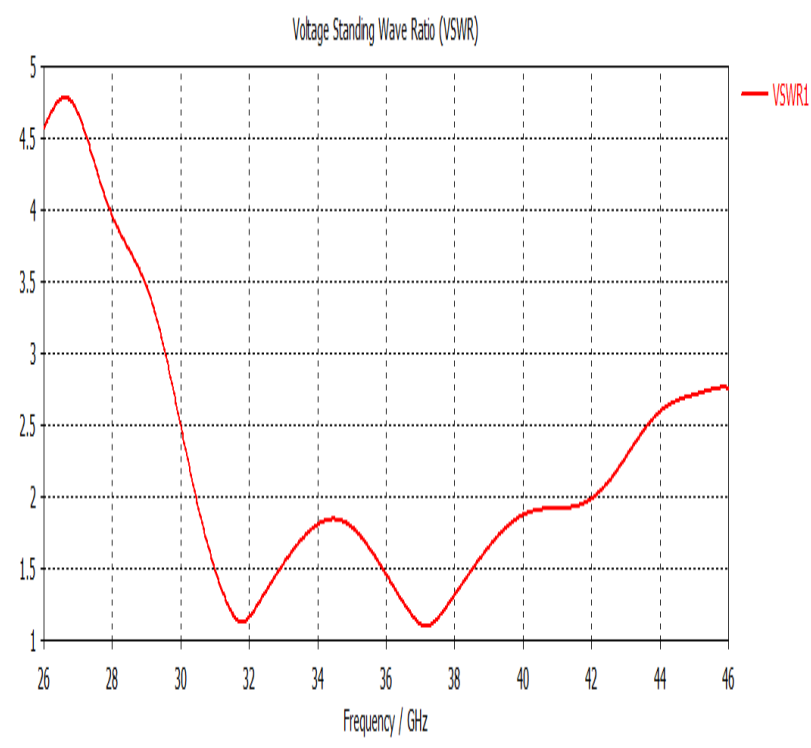

Figure 8. Simulated VSWR for the proposed antenna

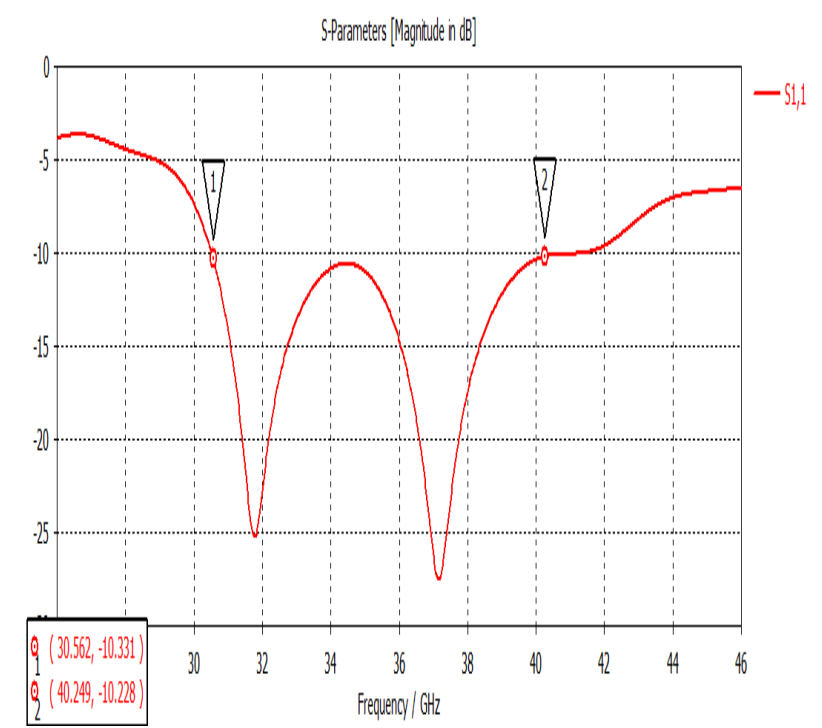

Figure 9. Simulated reflection coefficient (return loss for the proposed I shaped DRA verses frequency
Voltage Standing Wave Ratio: The ratio of the voltage ratio (VSWR) is a good parity between the impending impedance. VSWR is set as SWR. The VSWR antenna does not come up sooner than before. VSWR and the same imbalance as is the case with the same thing.The image proposes the simulation voltage relationship simulation (VSWR).

We have highlighted the antenna VSWR with 2 full frequency $30.8 \mathrm{GHz}$ and $39 \mathrm{GHz}$.

Radiation directivity, gain patterns: The peak gain of antenna is $10.9 \mathrm{dbi}$ at $35.39 \mathrm{GHz}$. It is observed from figure that the simulated peak directivity is $10.2 \mathrm{dBi}$ at $31.5 \mathrm{GHz}$ frequency range. In addition to that a total efficiency of $72.88 \%$ is found at $31.5 \mathrm{Ghz}$ and $69 \%$ is found at $\mathrm{GHz}$.

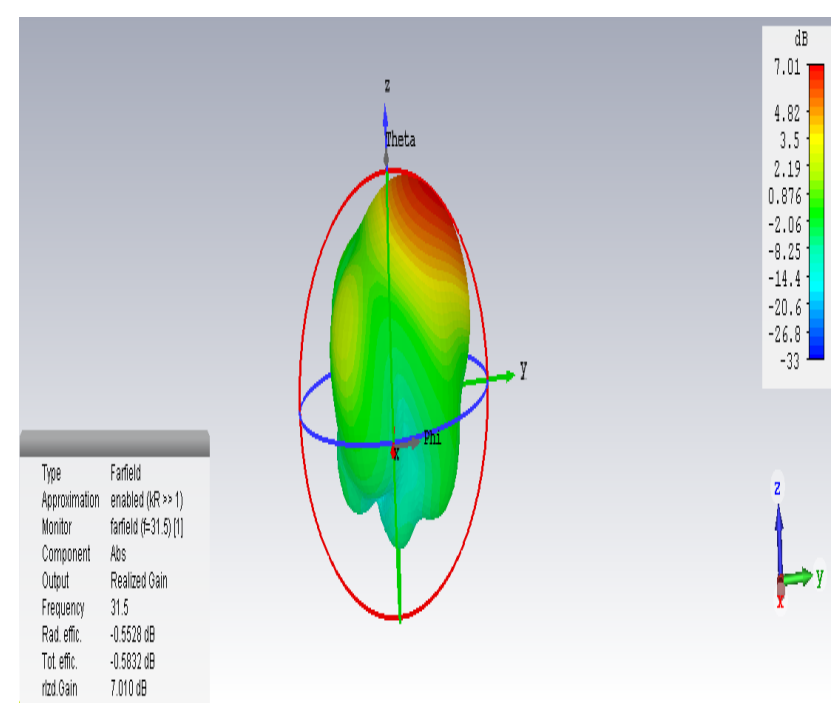

Figure 10. 3D view radiation pattern directivity of proposed DRA at frequency $31.5 \mathrm{GHz}$

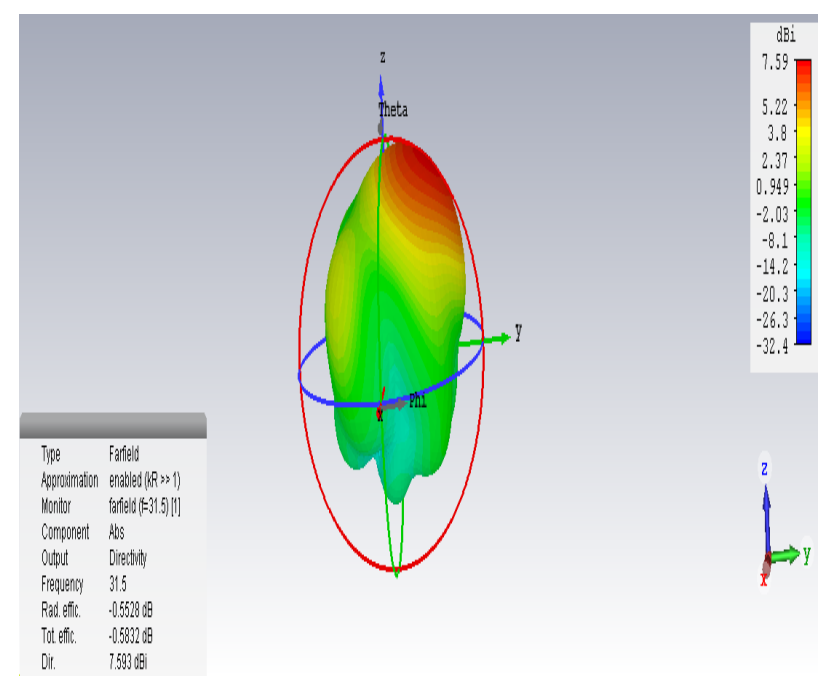

Figure 11. Directivity plot of the antenna

Relevance: The above mentioned result of s-parameter, simulated VSWR for the proposed antenna is relevant to our knowledge.

Effectiveness: The above mentioned result here is discussed about various aspects related to antenna design. Wireless communication in today's generation play vital role in all the areas. But maintaining a better communication is a bigger issue. The antenna system is a 
known technique which provides better information transmission and receiving.

Efficiency: After reviewing the researched paper techniques towards upgrading the overall performance of Micro strip patch antenna has been seen that there are many techniques with significant contribution.

However there are many pitfalls in the existing techniques too which give rise of various forms of unsolved questions.

\subsection{Challenges}

There is a little variation in the parameters studied in simulation process and results obtained after fabrication process. After fabrication of antenna, antenna radiates in the atmosphere. At the time of radiation of antenna, there are many metallic objects present in the environment which affects the propagation of electromagnetic waves. Due to these objects, reflections of EM waves take place. This leads to the variation in radiation pattern of antenna. Thus, we get variation in the antenna characteristics. The differences in the results after fabrication can also have a reason of manufacturing defects. It may contain impurities present in the material used for antenna fabrication. Also the environmental conditions like humidity; high temperature affects the charge distribution of patch which affects the characteristics of an antenna.

\section{Conclusion}

A dielectric antenna with slot has been modelled for ka-band applications. The proposed antenna parameters have been performed experimentally in CST MICROWAVE STUDIO and has been successfully validated. The effects of varying the distance between the two slots have been studied. The outcome of the proposed antenna makes it appropriate for use in ka-band applications (as it resonates between $26.5 \mathrm{GHz}$ and $40 \mathrm{GHz}$ ) which means for satellite communications.

At present, wearable textile antennas are under testing which can be used to constantly monitor the biometric data of the human body.

\section{Future Work}

Future work can be reached out by thinking about execution of improvement strategies or the DR radio wires. Some diagnostic models are required to get an unmistakable thought of attributes of radio wire. Great manufacture strategies ought to be pursued to limit the deliberate mistakes. Further work can be completed for various applications with various DRA structures and with various dielectric materials to fulfill the developing needs in the field of media transmission in present pattern.

\section{References}

[1] C. A. Balanis, "Antenna Theory, Analysis and Design”, John Wiley and Sons, 1997.

[2] K.L. Wong," Compact and Broadband Microstrip Antennas", John Wiley and Sons, 2002.

[3] Zhi Ning Chen and Michael Y. W. Chia, "Broadband Planar Antennas Design and Applicat ions", John Wiley \& Sons Inc., 2009

[4] Alejandro, A.-Z. (2008). Antennas and Propagation for Wireless Communication Systems. John Wiley \& Sons, Hoboken.

[5] N. Ghassemi, J. Rashed-Mohassel, and M. H. Neshati, "Microstrip Antenna Design for Ultra Wideband Application by Using Two Slots" Progress In Electromagnetics Research Symposium, pp. 169-171, March. 2015.

[6] S. A. Long, M. W. McAllister and L. C. Shen, "The resonant cylindrical dielectric cavity antenna", IEEE Trans. Antennas Propagat., vol. 31, pp. 406-412, May 1983. 